\title{
ODAR: AN ON-THE-FLY DAMAGE ASSESSMENT AND REPAIR SYSTEM FOR COMMERCIAL DATABASE APPLICATIONS
}

\author{
Pramote Luenam, Peng Liu \\ Dept. of Info. Systems, UMBC, Baltimore, $M D$
}

\begin{abstract}
This paper presents the design and implementation of an on-the-fly damage assessment and repair tool for intrusion tolerant commercial database applications, called ODAR. ODAR is a COTS-DBMS-specific implementation of a general on-the-fly damage assessment and repair approach developed by P. Ammann, S. Jajodia, and P. Liu in [8]. The general approach, given a set of malicious transactions reported by an intrusion detector, locates and repairs the damage caused by each malicious transaction on the database, along with the damage caused by any benign transaction that is affected, directly or indirectly, by a malicious transaction. The general approach locates and repairs damage on-the-fly without the need to periodically halt normal transaction processing. In this paper, the development of the first ODAR prototype, which is for Oracle Server 8.1.6, is discussed. ODAR uses triggers and transaction profiles to keep track of the read and write operations of transactions, locates damage by tracing the affecting relationships among transactions along the history, and repairs damage by composing and executing some specific UNDO transactions. ODAR is transparent to on-going user transactions and very general. In addition to Oracle, it can be easily adapted to support many other database application platforms such as Microsoft SQL Server, Sybase, and Informix. To our best knowledge, ODAR is the first tool that can do automatic on-the-fly damage assessment and repair for commercial database applications.
\end{abstract}

Key words: damage assessment, damage repair, database security, intrusion tolerance 


\section{INTRODUCTION}

Database security concerns the confidentiality, integrity, and availability of data stored in a database. A broad span of research from authorization $[3,4,5]$, to inference control [1], to multilevel secure databases [6,7], and to multilevel secure transaction processing [2], addresses primarily how to protect the security of a database. One critical step towards attack resilient database systems is intrusion detection (ID). ID protects systems by rejecting the future access of detected attackers. However, ID makes the system attack-aware but not attack-resilient, that is, ID itself cannot maintain the integrity and availability of the database in face of attacks.

To overcome the inherent limitation of ID, a broader perspective is introduced, saying that in addition to detecting attacks, countermeasures to these successful attacks should be planned and deployed in advance. In the literature, this is referred to as survivability or intrusion tolerance. In this paper, we will address a critical database intrusion tolerance problem beyond ID, namely attack recovery, and presents the design and implementation of an on-the-fly attack recovery tool, called ODAR. ODAR is a COTS-DBMSspecific implementation of a general on-the-fly damage assessment and repair approach developed by P. Ammann, S. Jajodia, and P. Liu in [8]. In this paper, the ODAR prototype, which is for Oracle Server 8.1.6, is discussed. ODAR is very general. It can be easily adapted to support many other DBMS. To our best knowledge, ODAR is the first tool that can do automatic on-the-fly damage assessment and repair for commercial database applications. We have tested ODAR using simulated data. The results show that ODAR causes very little performance penalty, is practical, and can be used to effectively help provide "data integrity" guarantees to arbitrary commercial database applications in face of attacks.

\section{ON-THE-FLY ATTACK RECOVERY APPROACH}

This section provides a simple introduction to our approach.

\section{- Affecting Relationships among Transactions}

In this approach, a database is a set of data items handled by transactions. A transaction is a partial order of read and write operations that either commits or aborts. The execution of a set of transactions is modelled by a structure called a history. In a history, a transaction $T_{i}$ is dependency upon another transaction $T_{j}$ if there exists a data item $x$ such that $T_{i}$ reads $x$ after $T_{j}$ updates it, and there is no transaction that updates $x$ between the time $T_{j}$ updates $x$ and $T_{i}$ reads $x$. The size of the intersection of $T_{j}$ 's write set and 
$T_{i}$ 's read set is called the dependency degree of $T_{i}$ upon $T_{j}$. The dependent upon relation indicates the path along which damage spreads. In particular, if a transaction which updates $x$ is dependent upon a malicious transaction which updates $y$, we say the damage on $y$ spreads to $x$, and we say $x$ is damaged. Moreover, a transaction $T_{u}$ affects $T_{v}$ if the ordered pair $\left(T_{v}, T_{u}\right)$ is in the transitive closure of the dependent upon relation. If malicious transaction $B_{i}$ affects an innocent transaction $G_{j}$, the damage on $B_{i}$ 's write set will spread to $G_{j}$ 's write set. If an innocent transaction $G_{j}$ is not affected by a malicious transaction, $G_{j}$ 's write set is not damaged. Therefore, the damage will be repaired if we back out every malicious or affected transaction.

To illustrate, consider the history as follows. $B_{1}$ affects $G_{2}$ but does not affect $G_{l} . x$ and $y$ are damaged but $z$ is not. So if $B_{l}$ is malicious then backing out $B_{1}$ and $G_{2}$ can repair the damage caused on $x$ and $y$.

$H$ : $\left(B_{l}\right.$ reads $\left.x\right)\left(B_{l}\right.$ writes $\left.x\right)\left(B_{l}\right.$ commits) $\left(G_{l}\right.$ reads $\left.z\right)\left(G_{2}\right.$ reads $\left.x\right)\left(G_{I}\right.$ writes $z)\left(G_{2}\right.$ reads $\left.y\right)\left(G_{1}\right.$ commits) $\left(G_{2}\right.$ writes $\left.y\right)\left(G_{2}\right.$ commits)

- The Algorithm

The approach, given a set of malicious transactions (denoted B) and a log that records every read and write operation, scans the log to identify every innocent transaction that is affected by $\mathbf{B}$. When a malicious transaction is scanned, its write set will be marked dirty. When a committed innocent transaction is scanned, if it reads a dirty item, then its write set will be marked dirty and a specific UNDO transaction will be composed and executed. When a data item is cleaned by an UNDO transaction, it will not be marked dirty anymore. For every item that is updated by a transaction to be undone, if the item is not cleaned by any previous UNDO transaction, then a write operation that restores the item to the value before it was damaged will be added to the UNDO transaction.

- Termination Detection

Since the algorithm allows users to continuously execute new transactions as the damaged items are identified and cleaned, new transactions can spread damage if they read a damaged but still unidentified item. So we face two critical questions: (1) Will the repair process terminate? (2) If the repair process terminates, can we detect the terminations? The general approach clearly answers these two questions. First, when the damage spreading speed is quicker than the damage repair speed, the repair process may never terminate. When the damage repair speed is quicker, the repair process will terminate. Second, under the following conditions we can ensure that the repair process will terminate: (1) every malicious transaction is repaired; (2) no item is marked dirty; (3) further scans will not identify any new damage. 


\section{ODAR}

In this section, we will present the design of ODAR. ODAR consists of six major components: (1) The Triggers (2) The Intrusion Detector (3) The Mediator (4) The Read Log Generator (5) The Write Log Generator (6) The Repair Manager. In Figure 1 we illustrate the architecture of ODAR.

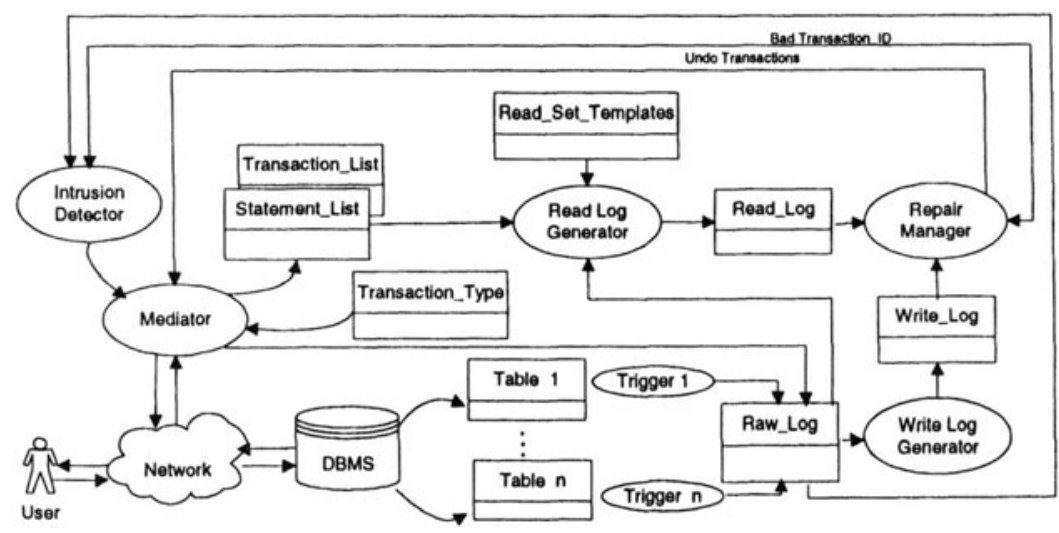

Figure 1. Design of ODAR

\subsection{Triggers}

We maintain read and write information by adding a trigger to each user table to collect the information about the write operations on that table. When a record is updated, deleted, or inserted, the corresponding trigger is executed and all the write operations are recorded into the Raw_Log table.

- Data Structure

- Raw_Log. This table keeps records of every write operation. Each record has 5 fields: (1) Transaction_ID that uniquely identifies a transaction; (2) Item_ID, a composite field, which consists of the Table_Name, Record_ID, and Field_Name; (3) Old_Value which keeps the item's value before it was modified; (4) New_Value which keeps the new item's value (5) Op_Type that indicates the categories of the command. The possible values include 'Insert', 'Update', 'Abort', and 'Commit'.

- An Example

To illustrate, we give an example to show how the trigger works. In this example, ODAR is applied to a banking application. Suppose we have a transaction for updating the customer account balance. The transaction 
contains 2 operations: an update and an insert. The update operation changes the customer account balance from $\$ 1,500.00$ to $\$ 2000.00$. Then, the insert operation records the user transaction into the Transactions table. In this example, writes information consists of the user transaction ID, Account_ID, Amount, Post_Date, and Teller_ID. This information is extracted from the transaction and inserted into the Raw_Log as shown in Figure 2.

Transection_D
4.91 .6240
4.91 .6240
4.91 .6240
4.91 .6240
4.91 .6240
4.91 .6240

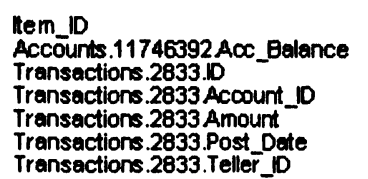

$\begin{array}{lll}\text { New_Value } & \text { Old_Value Op_Type } \\ 1500.00 & 2000.00 & \text { Ipdete } \\ & 2833 & \text { insert } \\ & 11746392 & \text { Insert } \\ & 500.00 & \text { Insert } \\ & 21-\text { Mar-00 } & \text { insert } \\ & \text { Teller-1 } & \text { insert }\end{array}$

Figure 2. Records in the Raw_Log generated by the trigger

\subsection{Intrusion Detector}

The Intrusion Detector has a responsibility to detect and report malicious transactions to the Repair Manager. It uses the trails kept in the Raw_Log and some other relevant proofs to identify bad transactions. If a bad transaction is active when being identified, the transaction will be aborted and any further malicious transactions submitted by the malicious user will be rejected. However, if a bad transaction is already committed when being identified, the bad transaction's identifier will be sent to the Repair Manager.

\subsection{Mediator}

The Mediator is responsible for maintaining read and write information. The main functions include: (1) Providing services to clients for accessing the database; (2) Capturing information about transactions' behavior. The information is then kept in the Statement_List and the Transaction_List; (3) Identifying the type of each in-coming transaction. This function provides support for capturing read operations. The Mediator identifies the type of a transaction by matching the transaction's statements with the transaction patterns kept in the Transaction_Type table.

- Data Structures

- Transaction_List table and Transaction_Type table. The transaction_list keeps some important information about transactions. The information includes the Transaction_ID, transaction status, and transaction type. The Mediator records log into the table when specific events occur in the transaction. The events include 'Start,' 'Commit,' and 'Abort.' The Transaction_Type keeps (1) transaction patterns that are used for 
matching operation and (2) the transaction type that is used to indicate the particular type of the transaction.

- Statement_List. This table records every client's SQL statement, which is sent to the database including the particular type and sequence.

- An Example

As described earlier, the Mediator is responsible for recording the read and write information into the Transaction_List and Statement_List (see Figure 4, 5). In addition, after a transaction is committed, the Mediator inserts a 'Commit' record into the Raw_Log (see Figures 6).

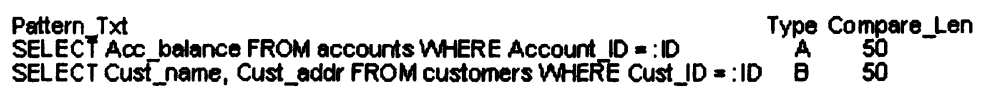

Figure 3. Example of a record in the Transaction_Type

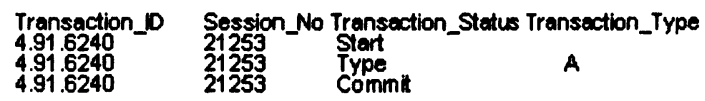

Figure 4. Example of records in the Transaction_List maintained by the Mediator

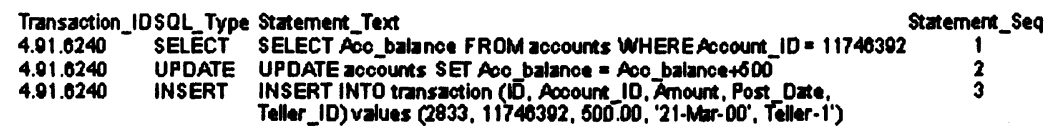

Figure 5. Example of records in the Statement_List maintained by the Mediator

\begin{tabular}{|c|c|c|c|c|}
\hline $\begin{array}{l}\text { Transaction_ll } \\
4.91 .6240 \\
4.91 .6240 \\
4.91 .6240 \\
4.91 .6240 \\
4.91 .6240 \\
4.91 .6240 \\
4.91 .6240\end{array}$ & $\begin{array}{l}\text { Hem_ID } \\
\text { Accounts.11746392 ACC_Balance } \\
\text { Transactions.2833.1D } \\
\text { Transactions. } 2833 \text { Account_10 } \\
\text { Transactions.2833 Amnount } \\
\text { Transactions.2833.Post_Date } \\
\text { Transactions.2833.Teller_ID }\end{array}$ & $\begin{array}{l}\text { New Value } \\
1500.00\end{array}$ & $\begin{array}{l}\text { Old Value } \\
2000.00 \\
2833 \\
11746392 \\
500.00 \\
21-\text { Mar-00 } \\
\text { Teller-1 }\end{array}$ & $\begin{array}{l}\text { Op_Type } \\
\text { Ipdete } \\
\text { Insert } \\
\text { Insert } \\
\text { Insert } \\
\text { Insert } \\
\text { Insert } \\
\text { Commit }\end{array}$ \\
\hline
\end{tabular}

Figure 6. Records in the Raw_Log after the 'Commit' operation is inserted

Suppose there are two types of transactions identified by 'A', and ' $B$ ' (see Figure 3) and the operation continues from the previous example in section 3.1. While the transaction is active, all SQL statements, including SELECT, UPDATE, and INSERT, will be recorded into the Statement_List (see Figure 5). In addition, the Mediator inserts three records about events of the transaction, 'Start', 'Commit' and the transaction type, into the Transaction_List table (see Figure 4). The transaction type is obtained by matching the first SQL statement of the transaction with the pattern stored in the Transaction_Type table. Before performing the pattern matching, the Mediator has to substitute the variable in the Pattern_Txt with the value 
extracted from the SQL statement. For example, the variable :ID will be substituted with '11746392' (Account_ID). After the Mediator successfully performs a matching, the transaction type will be searched out. In this example, the transaction type, obtained from the matching process, is ' $\mathrm{A}$ '.

\subsection{Read Log Generator}

The Read Log Generator maintains the Read_Log table to keep track of all the read operations. Once a 'Commit' operation is inserted into the Raw_Log table, the trigger on the Raw_Log will invoke the Read Log Generator. To capture read operations, First, the Mediator identifies the type of each in-coming transaction. This task can be done by matching each statement in a transaction with patterns kept in the Transaction_Type. Second, the Mediator records each SELECT statement and the relevant arguments in the Statement_List. The Mediator also keeps the type, status, and other information of the transaction in the Transaction_List. Third, the Read Log Generator uses the transaction type information and the arguments to materialize the relevant templates maintained in the Read_Set_Templates. Finally, the Read Log Generator retrieves read information from the Statement_List, and generates it into the Read_Log.

- Data Structures

- Read_Log. This table logs read operations of transactions. Each record has two fields: the Transaction_ID and the Item_ID.

- Read_Set_Templates. This table keeps the read operation patterns of transactions. Each record consists of: (1) Transaction_Type. The particular type of the transaction; (2) Statement_Seq. The sequence of the SELECT statements in the transaction; (3) Field_Seq. The sequence of fields in the SELECT statement; (4) Field_Name. The name of fields in the SELECT statement.

- An Example

Suppose this operation continues from the example in section 3.3. The Read Log Generator is invoked by the trigger on the Raw_Log, whenever a 'Commit' operation is inserted into the Raw_Log. The Transaction_ID ('4.91.6240') is passed to the Read Log Generator as an input parameter. By using the value, the Read Log Generator searches the log serial number (LSN) of the transaction from the Write_Log. However, the Write_Log is empty now. Therefore, the Read Log Generator has to generate the LSN itself. Suppose the generated value is 100 . With the $\mathrm{LSN}=$ ' 100 ' and the Op_type = 'Start', the Read Log Generator inserts the first record into the Write_Log (see Figure 8). 
In order to $\log$ the read operation, we need to know the value of the LSN, and the Item_ID. The Item_ID is composed of Table_Name, Record_ID, and Field_Name. The Table_Name and Record_ID can be extracted from the user SELECT statement while the Field_Name can be retrieved from the Read_Set_Templates by identifying the Transaction_Type and the Statement_Seq. The Transaction_Type can be obtained from the Transaction_List by searching with Transaction_ID $=$ '4.91.6240' and Transaction_Status = 'Type'. As a result, the Transaction_Type $=$ ' $A$ ' is returned (see Figure 4). For the Statement_Seq, we obtain the value from the Statement_Seq field in the Statement_List table. In this example, the Statement_Seq of the read operation $=$ ' 1 '. With the Transaction_Type = 'A' and Statement_Seq $=$ ' 1 ', the Read Log Generator gets all field name specified in the read operation from the Read_Set_Templates (see Figure 7). Finally, the Read Log Generator inserts the read information into the Read_Log table as shown in Figure 9.

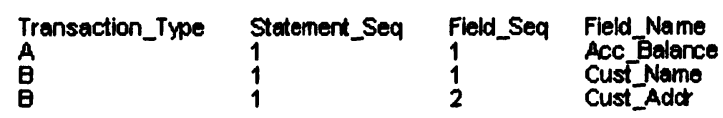

Figure 7. Example of records in the Read_Set_Templates

LSN Transaction_D Nem_ID New_Value Old_Value ${ }_{100}$ Sgant Type Time_Stamp
S1-Mar-00

Figure 8. Records in the Write_Log generated by the Read Log Generator

LSN Transaction_10 Item_10
101
4.91 .6240

Figure 9. Records in the Read_Log generated by the Read Log Generator

\subsection{Write Log Generator}

The Write Log Generator maintains the Write_Log table to capture write operations involved in an INSERT, UPDATE, or DELETE command. The Write Log Generator is invoked by the trigger associated with the Raw_Log table whenever a 'Commit' record is inserted into the Raw_Log table.

- Data Structure

- Write_Log Table. This table keeps records of every write operation. It has the same structure as the Raw_Log table except that it has the LSN column. The column keeps the LSN of a write record in a transaction. 


\subsection{Repair Manager}

The Repair Manager has a responsibility to perform on-the-fly damage assessment and repair. To do the job, the Repair Manager receives a message from the Intrusion Detector. The message contains the Transaction_ID, which is used to specify records of the malicious and affected transactions. All corresponding records, which contain the Transaction_ID, are retrieved from the Write_Log and the Read_Log table. For each data item in those records, the following operations are performed:

1. Keep the data item that might be marked as dirty items later. The suspect data item will be added to the tmp_item_set, if it is found in the cleaned_item_set, and the LSN of the item is greater than the LSN of the last current record in the Write_Log table.

2. Determine if a transaction has to be undone. A data item will be added to the tmp_undo_list, if (1) the in-repair transaction has read any data items in the dirty_item_set, or the cleaned_item_set, and (2) the LSN of the last record in the Write_Log is greater than the LSN of the read record.

3. Undo all changes made by a malicious or affected transaction. To perform the operation, all write records within the transaction are retrieved from the Write_Log table. The 'Insert' operation can be undone by deleting the corresponding records from the application table. While the 'Update' operation can be undone by rollback the current value in the application table to the old value that is kept in the Write_Log table.

4. Maintain the submitted_item_set by recording some details of every data item that's undo operation has been submitted to the Mediator but the item still has not been cleaned.

5. Maintain the dirty_item_set to keep data items that are marked as dirty.

- Termination Conditions

On-the-fly repair operations are performed repeatedly until the following termination conditions are satisfied: (a) every bad transaction has been undone; and (b) the dirty_item_set and the tmp_undo_list are empty; and (c) the LSN of each item stored in the cleaned_item_set is not less than the LSN of the next Write_Log entry to scan.

- Data Structure

The Repair Manager uses the following major data structures:

- tmp_item_set, an array of records that is used to keep information about an item (i.e. Item_ID) that may have been damaged.

- cleaned_item_set, an array of records that is used to keep information about items that have been cleaned. Each record of the array has two 
fields: (1) LSN that denotes log serial number of the bottom record of the $\log$ at the time when the item is cleaned; (2) Item_ID.

- dirty_item_set. This array keeps information of every data item that is marked as dirty. Each record of the array contains the Item_ID field.

- tmp_undo_list. This array keeps information about every in-repair transaction (i.e. Transaction_ID) that has read some data items in the dirty_item_set or satisfies others criteria which have been discussed in the previous section.

\section{TESTING ODAR}

In this section, we present the testing results of three experiments designed to examine the performance of ODAR. In each experiment, we measure the performance at two stages of databases -- busy and not busy. In busy status, the database was rarely idle. We simulated the busy status by running ten concurrent users. Each user continuously accessed the database at the same time. Each transaction made by a user is composed of several UPDATE and SELECT statements. However, these transactions only increase workload for database and do not generate any logs into our system.

For our testbed, we use Oracle 8.1.6 Server to be the underlying DBMS. The Oracle Server, the Mediator, and the Intrusion Detector are running on a DELL dual PIII 800Mhz PC with 512MB memory. The Repair Manager and the transaction simulator are running on a Gateway PC with a PIII $600 \mathrm{Mhz}$ and 128MB memory. These two PCs run Windows NT 4.0 and are connected by a $10 / 100 \mathrm{Mbps}$ switch LAN, however, the speed to the DELL Database Server is only 10Mbps. The current version of ODAR has around 20,000 lines of C++ and Oracle PL/SQL code. Each component of ODAR is implemented as a set of $\mathrm{C}++$ objects that have a couple of CORBA calling interfaces through which other components can interact with the component and the reconfiguration can be done. We use ORBacus V4.0.3 as the ORB.

\section{- Experiment I: Average Repair Time vs. Dependency Degree}

The objective of this experiment is to study the effects of changing the value of dependency degree to the average delay for cleaning damaged items. In this test, we simulate a sequence of transactions with a specific probabilistic dependency degree. Each dependency degree number denotes size of the intersection of a transaction's write set and its previous adjacent transaction's read set.

\section{- Experiment I: in Not Busy Database}

Various simulation parameters are specified in this experiment. First, five concurrent users are simulated to launch the transactions. The database 
contains 10,000 records. Each record contains 140 bytes. Three types of transactions are simulated: large, medium, and small. A large size transaction is composed of 15 read operations and 10 write operations. A medium size transaction is composed of 8 read operations and 4 write operations. A small size transaction is composed of 5 read operations and 2 write operations.

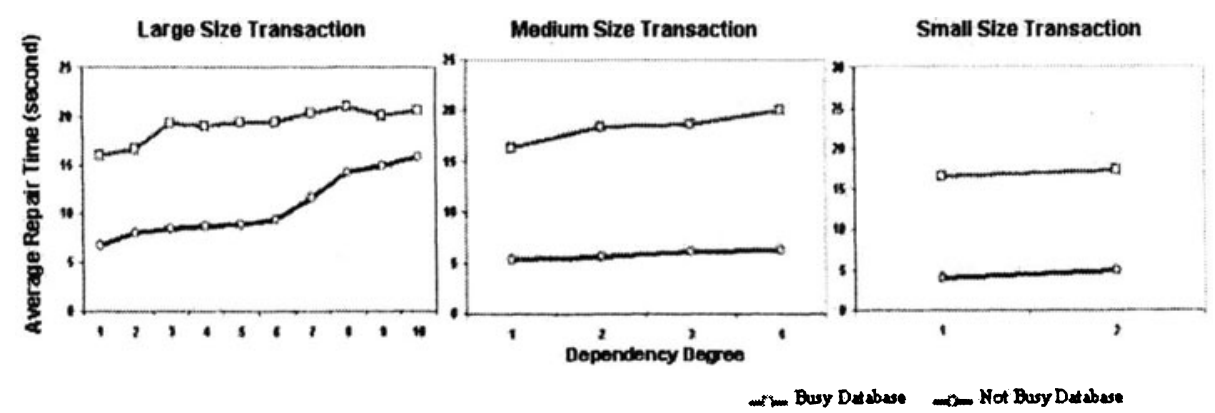

Figure 10. Average Repaired Time vs. Dependency Degree

The average values of the average repair time over dependency degree are plotted in graphs shown in Figure 10. Results show that the dependency degree affects system performance. For all three sizes of transactions, high dependency degree leads to high average repair time. The higher dependency degree increases the possibility of affected transactions. Consequently, the increase in the number of affected transactions increases the average repair time. Additionally, at the same dependency degree, performance suffers least when processing the small size transactions. Comparing the slope between three transaction sizes, the slope of the large size transaction is steepest. The reason is that more statements are needed to repair large size transactions. In addition, high average repair time for large size transactions may be due to data concurrency. Since we simulate this experiment with five concurrent users, there will be more chances for large size transactions when some records are simultaneously accessed by many transactions.

\section{- Experiment I: in Busy Database}

In this experiment, we measure the performance over dependency degree while the database is busy. Parameters used in this experiment are set to the same value as the previous one. Results are shown in Figure 10.

Compared to the previous experiment in the not busy database, the average repair time increases $70-150 \%, 130-160 \%$, and $145-165 \%$ for the large size transactions, medium size transactions, and small size transactions respectively. From the results, the performance is affected most by the small size transactions. The slope of the large size transactions is steepest among 
three sizes of transactions. The results support the previous finding that the higher dependency degree increases the possibility of affected transactions in large size transactions than that in medium and small size transactions.

- Experiment II: Average Repair Time vs. the Number of Transactions

The objective of this experiment is to test the Repair Manager's performance by measuring the average of time used for cleaning damaged items regarding several numbers of transactions. We measure the performance in two statuses of databases - busy and not busy.

- Experiment II: in not Busy Database
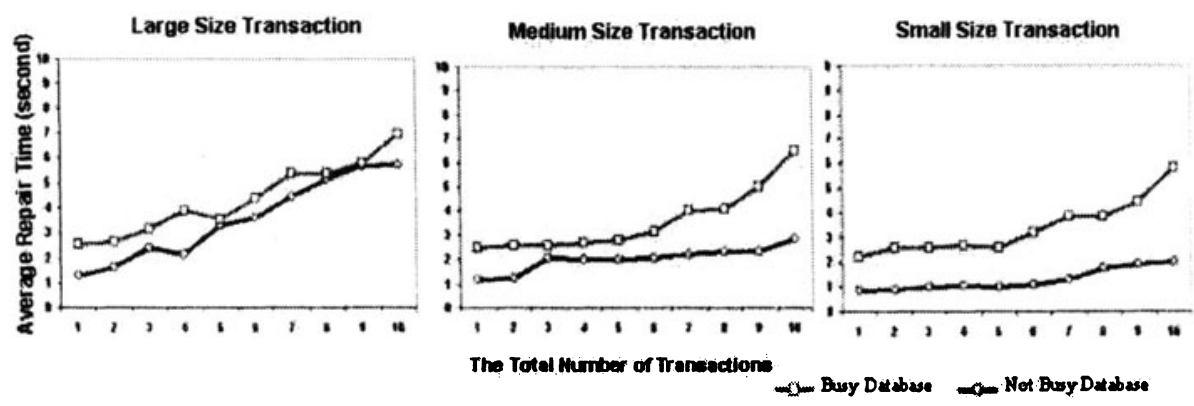

Figure 11. Average Repair Time vs. the Total Number of Transactions

The simulation parameters, used in this experiment, are set to the same value as the previous experiment except that we simulate only one concurrent user and we set the value of the dependency degree to 2 . Results (see Figure 11) show that the performance of the repair process is reduced as the total number of transactions is increased. For all sizes of transactions, high total number of transactions leads to high average repair time. This is because higher numbers of transactions increase the workload of the Repair Manager since the number of statements to be processed is increased.

Additionally, at the same total number of transactions, performance suffers least when processing the small size transactions. When comparing the slope between three transaction sizes, we can see that the slope of large size transactions is steepest among the three sizes of transactions. The higher number of transactions increases the possibility of affected transactions in large size transactions than that in medium and small size transactions.

- Experiment II: in Busy Database

In this experiment, we measure the performance over the total number of transactions when the database is rarely idle. All parameters are set to the same value as the previous experiment. Results (see Figure 11) show that the repair process, in the busy database, takes more average repair time than the not busy database. Average repair time increases 2-95\%, 25-130\% and 
$120-190 \%$ for large size transactions, medium size transactions, and small size transactions respectively. From the results, the Repair Manager's performance is affected most for small size transactions.

\section{- Experiment III: Impact on Throughput}

The objective of this experiment is to evaluate the impact of ODAR on the throughput of transaction processing. This test is performed by comparing the average time used for executing a single transaction when ODAR is deployed with the average time when ODAR is not enforced. Note that the average time used for executing a transaction represents the throughput of a database server.

\section{- Throughput in not Busy Database}

In this experiment, we measure the average repair time while no other transactions are processed except the simulated transactions. We simulate two types of transactions - large and small. All parameters, except the dependency degree, are set to the same value as the previous experiment. The average values of the average repair time when ODAR is/is not deployed over dependency degree are shown in Figure 12.

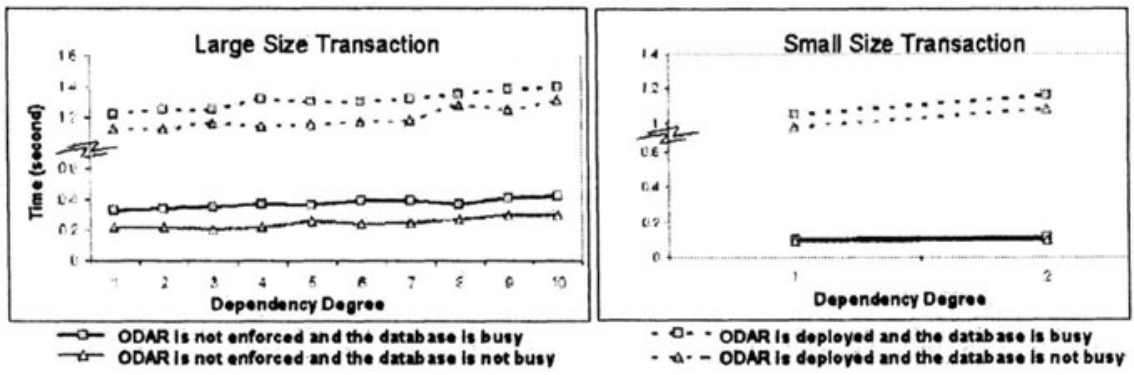

Figure 12. Impact on Throughput

Results indicate that ODAR affects performance of the system. The average time used for executing a transaction increases between $37-68 \%$ for large size transactions, and $11-20 \%$ for small size transactions. This is because large size transactions have much more read and write statements to be processed by the Mediator and the Repair Manager. Therefore, large size transactions have more performance penalty than small size transactions have. However, in real world applications where several read and write statements are usually combined into a single SQL statement, the response time delay should be much smaller than the results here.

\section{- Throughput in Busy Database}

In this experiment, we study the impact of ODAR on the average transaction execution time. Results are shown in Figure 12. Similar to the 
pervious experiment, performance drops when ODAR is deployed. Results show that, in the busy database, the average time used for creating a transaction increases for all sizes of transactions. It increases $30-50 \%$ for large size transactions and $10-30 \%$ for small size transactions. The results support the previous finding that large size transactions have more performance penalty than small size transactions have.

\section{CONCLUSION}

In this paper, we present the design and implementation of ODAR, an onthe-fly damage assessment and repair tool for intrusion tolerant commercial database applications. The first prototype of ODAR is tested using simulated data. The results show that the impact of ODAR on the performance of normal transaction processing is very small, which indicates that ODAR can be a practical solution towards providing "data integrity" guarantees to arbitrary commercial database applications in face of attacks.

\section{ACKNOWLEDGEMENT}

Luenam and Liu were supported by the Defense Advanced Research Projects Agency (DARPA) and Air Force Research Laboratory, Air Force Material Command, USAF, under agreement number F30602-00-2-0575.

\section{REFERENCES}

[1] M. R. Adam. Security-control methods for statistical database: A comparative study. ACM Computing Surveys, 21(4), 1989.

[2] V. Atluri, S. Jajodia, and B. George. Multilevel Secure Transaction Processing. Kluwer Academic Publishers, 1999.

[3] P. P. Griffiths and B. W. Wade. An authorization mechanism for a relational database system. ACM Transaction on Database Systems, 1(3):242-255, September 1976.

[4] S. Jajodia, P. Samarati, V. S. Subrahmanian, and E. Bertino. A unified framework for enforcing multiple access control policies. In Proceedings of ACM SIGMOD International Conference on Management of Data, pages 474-485, May 1997.

[5] F. Rabitti, E. Bertino, W. Kim, and D. Woelk. A model of authorization for nextgeneration database systems. ACM Transaction on Database Systems, 16(1):88-131, 1994.

[6] R. Sandhu and F. Chen. The multilevel relational (mlr) data model. ACM Transactions on Information and Systems Security, 1(1), 1998.

[7] M. Winslett, K. Smith, and X. Qian. Formal query languages for secure relational databases. ACM Transactions on Database Systems, 19(4):626-662, 1994.

[8] P. Ammann, S. Jajodia, and P. Liu. Recovery from malicious transactions. Technical report, George Mason University, Fairfax, VA. Under Review for Journal publication. 